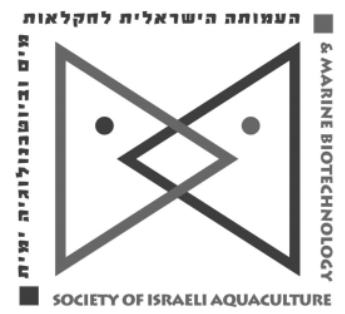

Published as an open-access journal by the Society of Israeli Aquaculture \& Marine Biotechnology (SIAMB).

To read papers free of charge, please register online at http://www.aquaculturehub.org/group/israelijournalofaquaculturebamidgehija The sale of IJA papers is strictly forbidden

\title{
Improved Sampling of Hemolymph and Screening of Anti-Coagulants of Hemocytes in the Snail Babylonia areolata
}

\author{
Guilan $\mathrm{Di}^{1}{ }^{1}{ }^{2}$, Guorong Zhu ${ }^{1}$, Yanfei $\mathrm{Li}^{1}$, Caihuan $\mathrm{Ke}^{2}$, \\ Zhaoxia Zhang ${ }^{*}$ \\ ${ }^{1}$ College of Fisheries, Henan Normal University, Xinxiang, 453007, China \\ ${ }^{2}$ College of Ocean and Earth Sciences, Xiamen University, Xiamen 361005, China
}

Keywords: anti-coagulant; Babylonia areolata; hemocyte

\begin{abstract}
Our aim was to improve sampling of hemolymph from the snail Babylonia areolata in order to evaluate the physiological and immune capacities of hemocytes in aquaculture. We also identified appropriate types of hemolymph anti-coagulants for $B$. areolata. Hemolymph samples were collected using an improved foot plantaris puncture method. We screened five types of anticoagulants from Penaeid shrimp (A), marine decapods (B), abalone (C), and oyster (D), as well as a home-made anti-coagulant (E), to act against Babylonia areolata hemocytes on the basis of cell death rate and hemocyte aggregation. We improved the former foot plantaris puncture method and identified the home-made anti-coagulant as the best anti-coagulant from the five which we tested.
\end{abstract}

* Corresponding author: Zhaoxia Zhang. Tel./fax: +86 592 2187420, email:

Zhaoxia_Zhang1@163.com 


\section{Introduction}

Babylonia areolata is a commercially important aquaculture species cultured along the southeast coast of mainland China. Due to its rich nutrition, rapid growth, and pleasing palatability, $B$. areolata has been identified one of the most important marine gastropods for human consumption (Fu et al., 2017; Lü et al., 2016). In China, especially in Hainan Province $B$. areolata was cultured in an area of over approximately 450000 square metres in 2013 and generated approximately RMB 250 million yuan (Fu et al., 2017; Lü et al., 2016). However, $B$. areolata production has decreased drastically in recent years because of the increased incidence of bacterial diseases such as vibriosis, proboscis intumescence disease, and shell cast disease (Feng et al., 2008). Thus, production of disease-resistant animals is crucial and depends on increasing understanding of marine invertebrate immunology. However, limited information is available about the cytoimmunity of marine gastropods compared with that of bivalve mollusks.

With less effective humoral factors in invertebrates, cellular immunity plays a more important role in invertebrates than in vertebrates. Hemocytes play a pivotal role in shellfish immunity (Soares-da-Silva et al., 2002). Observation of the function of hemocytes may help us understand the defensive reaction in immune systems of gastropods. However, mollusk hemocytes in vitro are easily aggregated by pseudopod interconnections after they leave their body (Auffret and Oubella, 2013; Zhou et al., 2017). In mollusks, hemocyte aggregation can induce clump formation which in turn affects hemostasis and wound healing. Hemocyte aggregation is difficult to reverse, making observation and research difficult ( $\mathrm{Li}, 2001)$, often compromising experimental designs. Another important consideration is the clotting progress. Hemocyte aggregation and clotting make it difficult to evaluate hemocyte immune capacities. Thus, hemocyte experiments use anti-coagulants to maintain the normal form of hemocytes in vitro and prevent hemocyte aggregation for a reasonable time. The anti-coagulant should not only prevent hemocyte aggregation but should also maintain cell integrity and hemocyte function and should also keep them as close as possible to their original physiological condition in vitro. However, the type and effective concentration of blood anti-coagulant that is the most suitable for gastropod hemolymph is still unknown. B. areolata (as in all snails) has an open circulation system, and its hemolymph contains hemocyanin. Appropriate anti-coagulants should be used in research on hemocyte functions of $B$. areolata. Almost all current hemocyte research in snails involves the use of buffer solution as an anti-coagulant (Sminia et al., 1979; Zhou et al., 1988) and the use of anticoagulants from shrimp and shellfish as references in experiments on other gastropod hemocytes. Until now, an anti-coagulant specific for gastropod hemocytes has been unavailable. Based on the few reports regarding gastropod hemolymph, the aim of this study was to improve sampling of hemolymph from the snail $B$. areolata, to select an anti-coagulant for the hemolymph, to investigate the effects of the five types of anticoagulants (one of them was home-made), and identify the appropriate type of hemolymph anti-coagulant for $B$. areolata.

Samples.

\section{Materials and Methods}

B. areolata adults $(3-4 \mathrm{~cm})$ were purchased from a farm in Dongshan, Fujian Province. The snails were maintained in a cement pond $(2 \mathrm{~m} \times 3 \mathrm{~m} \times 3)$ with flowing water (24$28^{\circ} \mathrm{C}$, salinity $\left.26-29, \mathrm{pH} 7.8-8.5\right)$. A layer of fine calcareous sand was added to the pond to allow burrowing. The snails were fed daily with oysters and chopped fresh fish.

Sampling of hemolymph.

B. areolata adults (3-4 cm shell length) were used for hemolymph sampling. Water adhering to the snail's surface and foot was removed and the snails were cleaned with absorbent paper. Hemolymph samples were obtained with an improved foot plantaris puncture method. Hemolymph was extruded using a $1.5 \mathrm{~mL}$ blue pipettes with the tip cut-off. This was inserted into the snail's foot while the snail's head and foot were forced to retract into the shell. Approximately $100 \mu \mathrm{L}$ of hemolymph was collected from each snail and the hemolymph was immediately transferred into $1.5 \mathrm{~mL}$ Eppendorf tubes containing the same quantity of anticoagulant to avoid hemocyte aggregation. The mixture was gently shaken to avoid clumping of hemocytes and then stored at $4^{\circ} \mathrm{C}$. 
Screening of anti-coagulants.

Anti-coagulant A was prepared in accordance with the Penaeid shrimp anti-coagulant formulation of Vargas-Albores et al. (1993). Anti-coagulant B was prepared in accordance with the Carpinus manas formulation of Söderhäll and Smith (1983). Anti-coagulant C was prepared in accordance with the abalone anti-coagulant formulation of Lebel et al. (1996). Anti-coagulant D was prepared in accordance with the oyster anti-coagulant formulation of Xue et al. (2001). Anti-coagulant E was prepared in-house. The homemade anti-coagulant solution (E) consisted of $2.05 \mathrm{~g}$ glucose, $0.8 \mathrm{~g}$ sodium citrate $\left(2 \mathrm{H}_{2} \mathrm{O}\right), 0.42 \mathrm{~g} \mathrm{NaCl}$, and $10 \mathrm{Mm}$ HEPES in $100 \mathrm{~mL}$ distilled water. $\mathrm{pH}$ was adjusted to 6.1 with $10 \%$ citric acid. The solution was sterilized at $121^{\circ} \mathrm{C}$.

Hemolymph from nine adult snails $(3-4 \mathrm{~cm}$ shell length) was pooled. Snail hemolymph and one type of anti-coagulant were mixed in equal amounts and stored at $4^{\circ} \mathrm{C}$ in an Eppendorf tube. Ten microliters of hemolymph mixture and an equal amount of $2 \%$ trypan blue solution were mixed on glass slides at different time intervals $(2,4,6,9$, $12,24,36$, and $48 \mathrm{~h}$ ). The mixture was observed through an oil immersion lens after 5 min to determine the number of dead cells per 100 cells at each time interval. Cell count was repeated three times. Hemocyte mortality rates at each time interval were calculated for each anti-coagulant. The state of hemocyte aggregation in each anti-coagulant was observed and recorded, including the numbers of cells in 10 fields of view for every sample and the average number of cells in each hemocyte cluster.

\section{Results}

Cell survival or death was determined by trypan blue staining. Live cells were white, whereas dead cells were blue (Fig. 1). After $2 \mathrm{~h}$, anti-coagulant $\mathrm{E}$ had the lowest hemocyte mortality rate of only $2.1 \%$. The hemocyte mortality rates in anti-coagulants $D, B$, and $A$ were $17.8 \%, 19.3 \%$, and $22.5 \%$, respectively. Anti-coagulant $C$ had the highest hemocyte mortality rate of $48.7 \%$. Hemocyte mortality rates increased in each anti-coagulant at time intervals up to $6 \mathrm{~h}$. After $9 \mathrm{~h}$, the hemocyte death rate for anticoagulant $\mathrm{E}$ was $9.3 \%$. It was the lowest among all five anti-coagulants for that time interval. For anti-coagulant B the hemocyte death rate was $42.2 \%$. The hemocyte mortality rates for anti-coagulants $D$ and $C$ reached $62 \%$ and $83.6 \%$, respectively; it was the highest at $84.8 \%$ for anti-coagulant $A$ and remained the highest up to the $24 \mathrm{~h}$ time interval for anti-coagulant A (Fig. 2). The hemocyte mortality rate for anti-coagulant $E$ somewhat increased but was the still lowest among all five anti-coagulants.

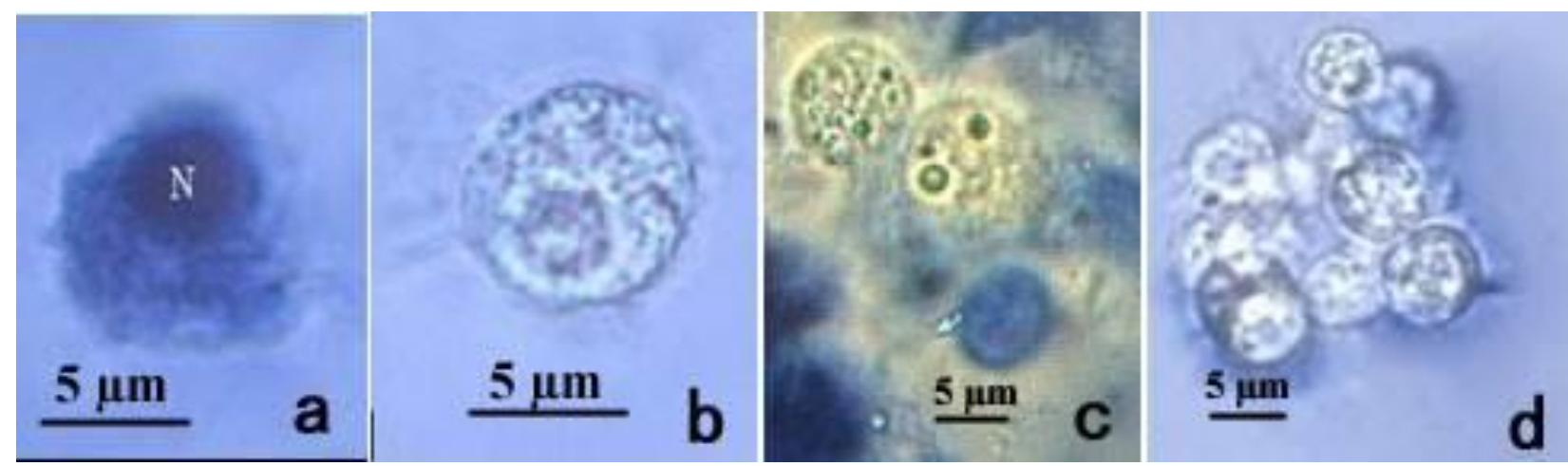

Fig. 1. Hemocytes in trypan blue reagent. (a), (b), and (c) show hemocytes with different responses to hemocyte staining with trypan blue reagent. Dead cells appear blue, of small volume, and with a large nuclear-cytoplasmic ratio, whereas live cells are transparent and colorless, of large volume, and with a small nuclear-cytoplasmic ratio. (a) shows a dead cell, while (b) shows a live cell. In (c), the colorless cells are alive, whereas the blue cells are dead. (d) shows a hemocyte cluster. $\mathrm{N}$ stands for the cell nucleus. 


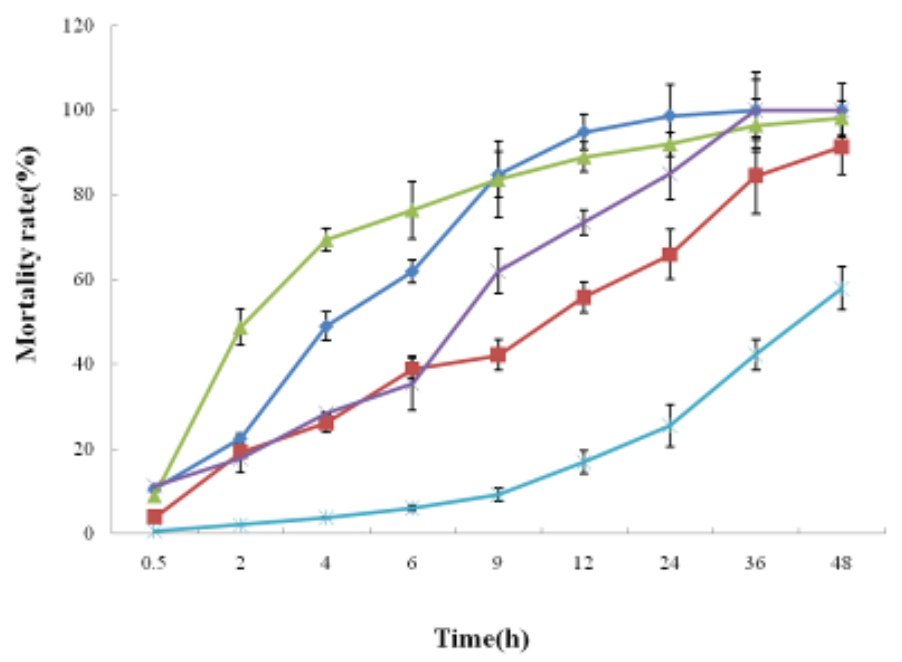

Fig. 2. Death rate of Babylonia areolata hemocytes in five anticoagulants.

$\mathrm{A}=$ Penaeid shrimp anti-coagulant;

$\mathrm{B}=$ marine decapod anti-coagulant;

$\mathrm{C}=$ abalone anticoagulant;

$\mathrm{D}=$ oyster anticoagulant; and $\mathrm{E}=$ home-made anticoagulant

No obvious trend was observed in the number of hemocyte clusters and the number of cells in each hemocyte cluster for the five types of anti-coagulants. The number of hemocyte clusters for anti-coagulant $\mathrm{C}$ and the average number of cells in each hemocyte cluster were lowest at the $48 \mathrm{~h}$ time interval. The average number of cells in each hemocyte cluster for anti-coagulant E was moderate among all five types of anticoagulant. The other three types of anti-coagulant showed irregular

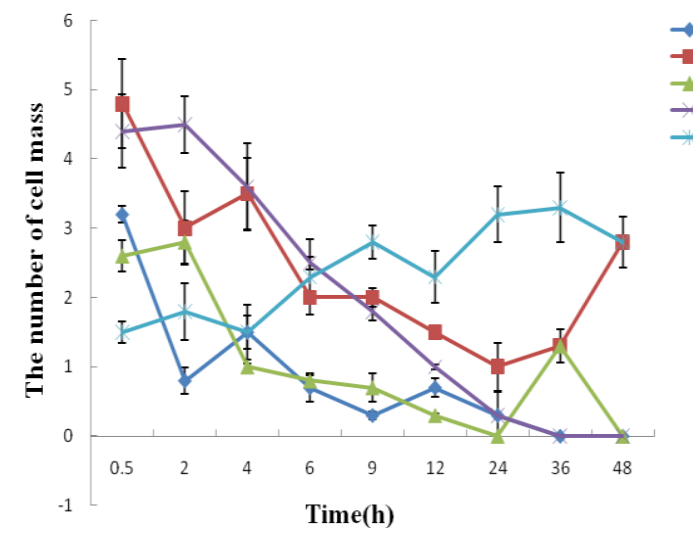
changes (Figs 3,4).

Fig. 3. Aggregated hemocyte cluster of Babylonia areolata hemocytes in five anticoagulants (Names of anti-coagulants as in Fig. 2.)

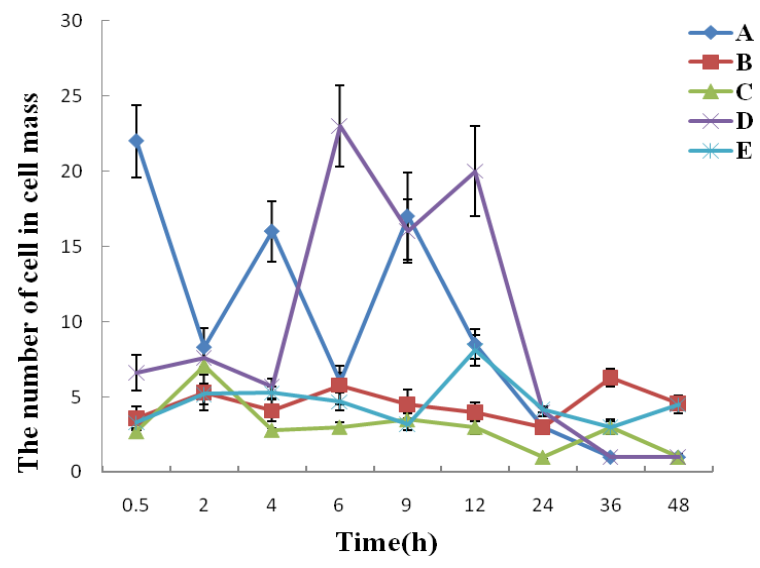

Fig. 4. Number of cells in the hemocyte cluster of Babylonia areolata hemocytes in five anticoagulants (Names of anti-coagulants as in Fig. 2.) 
In summary, hemocyte mortality rate was lowest for anti-coagulant E. Anti-coagulant $E$ exhibited the most even hemocyte distribution and most effective anti-coagulant action. Anti-coagulant $\mathrm{C}$ also had effective anti-coagulant action, while its hemocyte mortality rate was high.

Hemolymph collection method.

Discussion

Snail hemolymph collection methods include foot plantaris puncture (Sminia et al., 1979), capsular discission (Jeong and Heyneman, 1976), heart puncture (Cheng and Auld, 1977), head and foot flesh suction (Wang et al., 1994), and head and foot flesh extrusion (Tan et al., 2001). Each method has its particular advantages and disadvantages. While the foot plantaris puncture method is convenient and simple, hemolymph is sometimes wasted as the glutinous hemolymph easily sticks to the wall of the collecting tube and may block the tube nozzle due to the small dimensions of the pipette. The capsular discission method is an incision similar to the heart puncture method. It is possible to use both methods to collect high-quality hemolymph, however the accurate location of the heart must be determined before drilling a hole in the snail shell. The head and foot flesh suction method requires lengthy stimulation time, and only a very small quantity of hemolymph fluid is collected. The head and foot flesh extrusion method is applicable only for blood smears and is not recommended for hemolymph sampling.

In our B. areolata hemolymph collection experiment we used an improved foot plantaris puncture method. The principle of this method is similar to the head and foot flesh extrusion method. The sampled fluid, which is blue due to its ion content, is the same as the hemolymph in the lung's pulmonary vein in terms of unit volume and cell density, and is recognized as hemolymph fluid (Sminia, 1975). Instead of using a slender pipette as in the foot plantaris puncture method, we used pipette tips with cut-off heads which are convenient to use and prevent the hemolymph fluid from blocking the pipette nozzle.

Some of our experiences with hemocyte fluid collection are summarized below: The colorless, seawater-like fluid squeezed out from the gap between the shell and the foot when stimulating the foot plantaris with the tip of the liquid-transferring pipette could not be taken for hemolymph collection, however the slightly blue dense fluid subsequently extruded was hemolymph. In general, $90 \mu \mathrm{L}$ of hemolymph could be sampled from a snail of approximately $3.05 \mathrm{~cm}$ shell length and $6.12 \mathrm{~g}$ weight. To avoid waste and shortage of the hemolymph, the foot plantaris was not strongly stimulated during sampling, and the hemolymph was extracted slowly. The hemolymph would also merge with other fluids if the foot plantaris was stimulated too strongly or if the hemolymph was squeezed out too quickly. This phenomenon could affect the result of the experiment. Thus, we found that the foot plantaris should be stimulated gently with the tip of the liquid-transferring apparatus, and no squeezing should be used. These were our modifications and improvements on the former foot plantaris puncture method.

Anticoagulant screening.

Anti-coagulants are extremely important in hemocyte research. In hemocyte research failure is often due to lack of an appropriate anti-coagulant, resulting in hemocyte death or aggregation. In this study, we selected four anti-coagulants previously identified, and in addition we prepared an anti-coagulant (E). All five of the anti-coagulants used in this study were screened for cell mortality rate and aggregation with anti-coagulants recorded over different time intervals. The mortality rates of snail hemocytes were very high in the prawn (Vargas-Albores et al., 1993), abalone (Lebel et al., 1996), and oyster (Xue et al., 2001) anti-coagulants, and aggregation was extensive. However, the home-made anticoagulant $\mathrm{E}$ resulted in longer viability for snail hemocytes and greatly reduced aggregation. Consequently, we considered it to be the best anti-coagulant for hemocyte research of $B$. areolata. Based on these results we recommend the use of anti-coagulant $E$ in hemocyte immunology research of $B$. areolata. We do not recommend the use of 
anti-coagulants $A, B$, and $D$ because of their high mortality rates and poor anti-coagulant effects.

Results from this experiment supplied valuable experience for gastropod research and will benefit the development of snail hemocyte research, contribute to the knowledge of gastropod immunology, gain greater insight into the cytology-based mechanisms of immune defense and disease resistance of hemocytes in the future, and also provide reference data for $B$. areolata aquaculture. In addition, anti-coagulant $E$ also performed best in other hemocyte experiments on the abalone Haliotis diversicolor and Scylla serrata (unpublished data). Anti-coagulant $\mathrm{E}$ has the potential to become an ideal anticoagulant for marine gastropods and crustaceans.

\section{Conclusion}

We modified and improved the foot plantaris puncture method for hemolymph sampling. And succeeded in demonstrating that anti-coagulant E prepared in our laboratory was the most appropriate hemolymph anti-coagulant for Babylonia areolata.

\section{Acknowledgements}

This work was supported by NSFC (No. 31640085), the Basic and Frontier Technology Research Program of Henan Province (No. 152300410206), the Key Project of Science and Technology Research of Henan Provincial Department of Education (No. 14B240003), the Projects of the Science and Technology Department of Henan Province (182102110328).

\section{References}

Auffret M., Oubella R., Auffret M . and Oubella R, 1997. Hemocyte aggregation in the oyster Crassostrea gigas: In vitro measurement and experimental modulation by xenobiotics. Comp. Biochem. Physiol. A., 118(3): 705-712.

Cheng T.C. and K.R. Auld, 1977. Hemocytes of the pulmonate gastropod Biomphalaria glabrata. J. Invertebr. Pathol., 30: 119- 122.

Feng Y.Q., Zhou Y.C., Xie Z.Y., Pu L.Y., Lu N., Lin Z.Y., Zhou J.X. and X.Y. Fu, 2008. Studies on the techniques of healthy culture for Babylonia areolata. Chinese Fishery Modernization, 35: 39-41.

Fu J., Lü W., Li W., Shen M., Luo X., Ke C. and You W, 2017. Comparative assessment of the genetic variation in selectively bred generations from two geographic populations of ivory shell (Babylonia areolata). Aquac. Res., 48: 4205-4218.

Jeong K.H. and D. Heyneman, 1976. Leukocytes of Biomphalaria glabrata: Morphology and behavior of granulocytic cells in vitro. J. Invertebr. Pathol., 28: 357-362.

Lebel J.M., Giard W., Favrel P. and E. Boucaud-Camou, 1996. Effects of different vertebrate growth factors on primary cultures of hemocytes from the gastropod mollusc, Haliotis tuberculata. Biol. Cell., 86: 67-72.

Li Y, 2001. The effect of six anticoagulant commonly used to the Hemolymph of Macrobrachium nipponense. Chinese Reservoir Fisheries, 21: 41 - 42.

Lü W., Ke C., Fu J., You W., Luo X., Huang M. and Yu J, 2016. Evaluation of crosses between two geographic populations of native Chinese and introduced Thai spotted ivory shell, Babylonia areolata, in southern China. J. World. Aquacult. Soc., 47(4): 544-554.

Soares-Da-Silva I.M., Ribeiro J., Valongo C., Pinto R., Vilanova M., Bleher R. and J. Machado, 2002. Cytometric, morphologic and enzymatic characterisation of haemocytes in Anodonta cygnea. Comp. Biochem. Physiol. A. Mol. Integr. Physiol., 132: 541-553.

Söderhäll K. and V.J. Smith, 1983. Separation of haemocyte populations of Carcinus maenus and other marine decapods, and prophenoloxidase distribution. Dev. Comp. Immunol., 7: 229-239.

Sminia T, 1975. Structure and function of blood and connective tissue cell of the freshwater snail Lymnaea stagnalis. Ph. D. Thesis, Free University, Amsterdam, the Netherlands.

Sminia T., Van Der Knaap W.P.W. and P. Edelenbosch, 1979. The role of serum factors in phagocytosis of foreign particles by blood cells of the freshwater snail Lymnaea stagnalis. Dev. Comp. Immunol., 3: 37-44. 
Tan P., He C.H. and T.P. Gong, 2001. A sort of simple and effective method used in checking the hemocytes of Oncomelania hupensis. Chin. J. Schisto. Control., 13: 222223.

Vargas-Albores F., Guzmán-Murillo M.A. and J.L. Ochoa, 1993. An anticoagulant solution for haemolymph collection and prophenoloxidase studies of Penaeid shrimp (Penaeus californiensis). Comp. Biochem. Physiol., 106A: 299-303.

Wang X.Q., Qiu L.S., He Y.X. and S.P. Mao, 1994. Morphology and phagocytic activity of hemocytes of Oncomelania hupensis. Chinese Journal of Parasitology and Parasitic Diseases, 2: 100-103.

Xue Q.G., Renault T. and S. Chilmonczyk, 2001. Flow cytometric assessment of haemocyte sub-populations in the European flat oyster, Ostrea edulis, haemolymph. Fish. Shellfish. Immunol, 11: 557-67.

Zhou T., Yiang J.H. and M. Zhou, 1988. Karyotype analysis of Oncomelania hupensis. Chinese Journal of Zoology, 23: 6-8.

Zhou L., Yang A., Liu Z., Wu B., Sun X., Lv, Z., Tian J. and Du M, 2017. Changes in hemolymph characteristics of ark shell Scapharaca broughtonii dealt with Vibrio anguillarum challenge in vivo and various of anticoagulants in vitro. Fish. Shellfish. Immunol., 61: 9-15. 